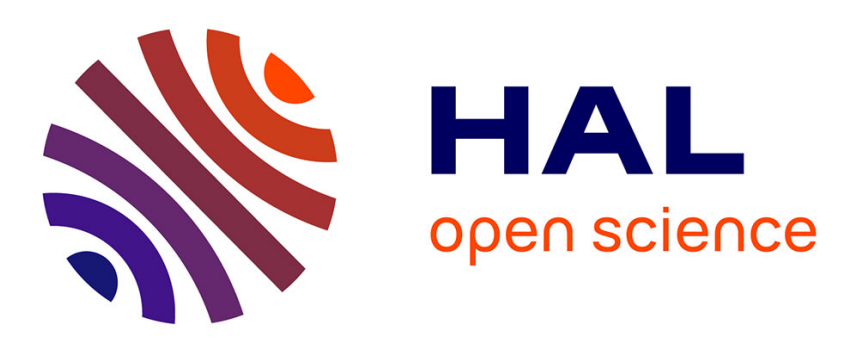

\title{
Enhanced evaporation from an oscillating liquid in a capillary tube
}

\author{
Denis Polezhaev, Paul Duru, Franck Plouraboué
}

\section{To cite this version:}

Denis Polezhaev, Paul Duru, Franck Plouraboué. Enhanced evaporation from an oscillating liquid in a capillary tube. International Journal of Heat and Mass Transfer, 2016, vol. 95, pp. 288-295. 10.1016/j.ijheatmasstransfer.2015.12.012 . hal-01252783

\section{HAL Id: hal-01252783 \\ https://hal.science/hal-01252783}

Submitted on 8 Jan 2016

HAL is a multi-disciplinary open access archive for the deposit and dissemination of scientific research documents, whether they are published or not. The documents may come from teaching and research institutions in France or abroad, or from public or private research centers.
L'archive ouverte pluridisciplinaire HAL, est destinée au dépôt et à la diffusion de documents scientifiques de niveau recherche, publiés ou non, émanant des établissements d'enseignement et de recherche français ou étrangers, des laboratoires publics ou privés. 


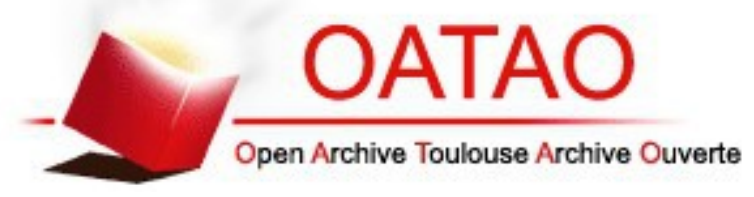

\section{Open Archive TOULOUSE Archive Ouverte (OATAO)}

OATAO is an open access repository that collects the work of Toulouse researchers and makes it freely available over the web where possible.

This is an author-deposited version published in : http://oatao.univ-toulouse.fr/ Eprints ID : 14612

To link to this article : DOI:10.1016/j.ijheatmasstransfer.2015.12.012 URL : http://dx.doi.org/10.1016/j.ijheatmasstransfer.2015.12.012

To cite this version : Polezhaev, Denis and Duru, Paul and Plouraboué, Franck Enhanced evaporation from an oscillating liquid in a capillary tube. (2016) International Journal of Heat and Mass Transfer, vol. 95. pp. 288-295. ISSN 00179310

Any correspondance concerning this service should be sent to the repository administrator: staff-oatao@listes-diff.inp-toulouse.fr 


\title{
Enhanced evaporation from an oscillating liquid in a capillary tube
}

\author{
D. Polezhaev ${ }^{a}$, P. Duru ${ }^{\text {b }}$ F. Plouraboué $e^{\mathrm{b}, \mathrm{s}, *}$ \\ ${ }^{a}$ Laboratory of Vibrational Hydromechanics, Perm State Humanitarian Pedagogical University, 24, Sibirskaya Str., 614990 Perm, Russia \\ ${ }^{\mathrm{b}}$ Université de Toulouse, INPT, UPS, IMFT (Institut de Mécanique des Fluides de Toulouse), 2 Allée Camille Soula, F-31400 Toulouse, France \\ ${ }^{\mathrm{c}}$ CNRS, IMFT, F-31400 Toulouse, France
}

\section{A R T I C L E I N F O}

\section{Article history:}

Received 25 June 2015

Received in revised form 4 November 2015

Accepted 2 December 2015

\section{Keywords:}

Vapour diffusion

Evaporation

Taylor's dispersion

Capillary tube

Oscillatory flow

\begin{abstract}
A B S T R A C T
Enhanced evaporation inside a capillary tube into which the liquid/gas meniscus oscillates is experimentally studied. It is found that the meniscus oscillation can markedly level-off the evaporation rate, while keeping an apparent diffusive behaviour. The apparent diffusive coefficient can reach a tenfold increase in the explored range of parameters. The dependence of the effect is studied by varying the capillary tube diameter, the frequency and the amplitude of the liquid oscillations. The parametric dependence of the apparent diffusive coefficient is well captured by the associated dimensionless Péclet number. A nice collapse of the experimental measurements consistent with a quadratic scaling with Péclet number is found. Such scaling is suggested by previous theoretical and experimental analysis associated with a Taylor dispersion transport mechanism. Nevertheless the prefactor of those theory is found to under-predict the observed effect by a factor three. This deviation from Taylor's dispersion driven transport predictions is discussed.
\end{abstract}

\section{Introduction}

In the present work, we study the evaporation of a volatile liquid contained in a vertical capillary tube, when oscillations of the liquid/gas interface meniscus are imposed. Evaporation is due to vapour diffusion through air, from the oscillating meniscus to the still ambient external air, where the mass fraction of the vapour is negligible. This problem is related to the classical Taylor's dispersion phenomenon, named after his discoverer [1]. The classical analysis of Taylor's dispersion deals with the enhanced dispersion of a tracer in a non oscillatory flow. When a fluid flows through a tube, a velocity profile develops in the transverse direction due to the no-slip boundary condition at the tube's edge. Since the resulting velocity profile is not uniform, over the cross-section of the tube the fluid move faster in the centre than near the edges. Such transverse gradient of the longitudinal velocity is the key ingredient for longitudinal dispersion to occur: a small plug of vapour in air which diffuses transversely from the centre to the edge will experience a strong longitudinal shear because a change in transverse position will translate into a change in longitudinal velocity. This mechanism explains how the apparent longitudinal

* Corresponding author at: Université de Toulouse, INPT, UPS, IMFT (Institut de Mécanique des Fluides de Toulouse), 2 Allée Camille Soula, F-31400 Toulouse, France.

E-mail addresses: polezhaev@pspu.ru (D. Polezhaev), duru@imft.fr (P. Duru), fplourab@imft.fr (F. Plouraboué) diffusion of vapour along the direction of air flow is enhanced. As a phenomenon, Taylor's dispersion resembles molecular diffusion, but it actually results from the interaction between transverse diffusion and velocity shear, and is therefore a function of the flow type and the channel geometry. Other factors, like flow dynamics and chemical reactions can produce additional effects to the dispersion. In laminar flow with molecular diffusivity $D$, the effective longitudinal diffusion coefficient can be shown to fulfil $D_{\text {eff }}=D\left(1+\gamma P e^{2}\right)$, where $P e \equiv\langle u\rangle d / D(\langle u\rangle$ is the average velocity over the tube cross-section) is the Péclet number and the parameter $\gamma$ depends on the shape of the channel cross-section and velocity profile [2]. Taylor [1] showed that for Poiseuille flow in a circular tube of diameter $d, \gamma=1 / 192$.

A similar effect occurs when the flow is oscillatory, as is the case in the present work. An oscillatory tube flow is characterised by the Womersley number $\alpha=\frac{d}{2}\left(\frac{\omega}{v}\right)^{1 / 2}$, where $v$ is the kinematic viscosity, $\omega$ the angular-frequency, and the Reynolds number $R e_{\delta}=\bar{u} \delta / v$, where $\bar{u}=2 b \omega$ is the cross-section average of the peak velocity amplitude which depends on the oscillation amplitude $b$ and $\delta=(2 v / \omega)^{1 / 2}$ is the Stokes-layer thickness. Experiments [3] have shown that such a flow is laminar when the Reynolds number is bellow a given threshold: $R e_{\delta}<500$. For $R e_{\delta}>500$, the core flow remains laminar while the Stokes layer becomes unstable during the deceleration phase of fluid motion. For increasing Reynolds number the turbulence is confined to an annular region which is a few times the Stokes-layer thickness near the wall. Thus, in this 


$$
\begin{aligned}
& \text { Nomenclature } \\
& A \equiv\left(2 D \rho_{v} / \rho_{l}\right) \\
& A_{e f f} \equiv\left(2 D_{e f f} \rho_{v} / \rho_{l}\right) \\
& \frac{d}{2}\left(\frac{\omega}{n u}\right) \\
& b \\
& \beta \equiv \delta_{f} / d \\
& D \\
& d \\
& D_{e f f} \\
& \delta \equiv(2 v / \omega)^{1 / 2} \\
& \delta_{f} \\
& D_{l} \\
& f \equiv 2 \pi / \omega \\
& \omega \\
& M \\
& \mu \\
& v \\
& P_{a} \\
& P e \equiv\langle u\rangle d / D \\
& R
\end{aligned}
$$

\section{$\omega$}

parameter quantifying the evaporation kinetic

parameter quantifying the evaporation kinetic, in the oscillatory case

Womersley number

amplitude of the imposed oscillation of the meniscus

dimensionless liquid film thickness

diffusivity of vapour (2-propanol) in air

tube diameter

effective diffusivity of vapour (2-

propanol) in air

Stokes layer thickness

liquid film thickness

self-diffusivity of (2-propanol) in liquid phase

frequency of the imposed oscillation of the meniscus

angular-frequency of the forcing stroke

molecular weight of 2-propanol

dynamic viscosity of the air

kinematic viscosity of the air

ambient air pressure

Péclet number

ideal gas constant $r$

$R e \equiv\langle u\rangle d / v$
$\operatorname{Re}_{\delta}$
$\rho_{l}$
$\rho_{v}$
$S \equiv \pi d^{2} / 4$
$\sigma \equiv v / D$
$T$
$t$
$T_{a}$
$u$
$\langle u\rangle \equiv \frac{2 \pi}{\pi d^{2}} \int_{0}^{d / 2} u(r) r d r$
$\bar{u} \equiv 2 b \omega$
$V_{\beta}$
$x_{e}$
$z$
$z_{0}$
$\gamma$

radial cylindrical coordinate associated with the tube

Reynolds number of the flow

Reynolds number of the flow based on $\delta$

mass density of the liquid 2-propanol

mass density of 2-propanol vapour

tube cross-section area

schmidt number

2-propanol imposed temperature

time

ambient air temperature

fluid longitudinal velocity

averaged longitudinal fluid velocity

cross section average of the peak velocity amplitude

fluid (2-propanol) volumetric thermal expansion coefficient

thermodynamical equilibrium mass fraction of 2-propanol-2 in air

longitudinal coordinate along the cylinder direction

initial meniscus location

Taylor dispersion parameter limit, the axial transport of a passive contaminant is compounded by the interaction of the inviscid core with the turbulent boundary layer. The convective and diffusive mechanisms in laminar oscillatory flows are the same as in steady flow but a wider variety of results are found. Shear-augmented axial dispersion in oscillatory flows has been studied in [4-10] among others. For low $\alpha$, the coefficient $\gamma$ is predicted to be $1 / 192[6]$ which is in agreement with the steady-flow result [2]. However, the experimental data in this range of $\alpha$ are rather scarce $[11,8,12]$.

In this paper, we specifically report the results of an experimental study of vapour enhanced-diffusion in laminar oscillatory flow of air inside a capillary tube at $\alpha \simeq 1$. The focus of the study is to measure the effective diffusion coefficient as a function of the amplitude and frequency of the oscillations and of the tube diameter.

Pulsed flow in pipes can indeed be found in various applied context such as Stirling engines and pulse tube cryocoolers for space applications, where heat transfer improvement has been reported [13]. In more academic contexts, using forced oscillations to enhance heat transfer has been demonstrated to be useful either in straight tubes $[14,15,13,16]$ or constricted ones [17]. Two reviews have emphasised the interest of oscillating flows for enhanced heat transfer in pipes and tubes [18,19]. A recent study has experimentally measured the temperature profile along longitudinal direction in a gas flow between two parallel plates, and confirm the interest of oscillation to enhanced transfert [20]. Nevertheless, even though such elementary pump system has been studied in the context of single phase flows, the interest of oscillating meniscus for heat transfer evaporation has not been quantitatively and precisely studied yet in simple configurations. The aim of this paper is to fill this gap in the litterature, with an emphasis on providing relevant dimensionless parameter to the transfer rate. In this paper we thus experimentally investigate how oscillating transfer can be interesting for enhanced evaporation inside a tube.

The paper is organized as follows, Section 2 is devoted the presentation of the experimental set-up. Section 3 presents the obtained results within a critical and synthetic manner. In Section 4 , we discuss the results in the light of theoretical predictions found in the litterature, as well as comparisons with previous experimental results.

\section{Experimental setup and protocol}

\subsection{Experimental setup}

The experimental setup is shown in Fig. 1. It consists of a syringe filled with fluid and connected to a long borosilicate glass capillary tube (Fig. 1a). The tube diameter, $d$, varies from 0.70 to $2.05 \mathrm{~mm}$. The syringe is placed inside a bronze cavity to manage the temperature of the working fluid (see below). In the experiments, we use three-component syringes with rubber gasket. The flexible gasket plays the role of a membrane to generate the fluid oscillations. The membrane is driven by a rod attached to the centre of an acoustic louder. The handling of the acoustic louder is carried out by a digital generator PGSF-052 and an amplifier Digisynthetic DP3200. The electrical signal of the generator is transmitted to the amplifier and then to the acoustic louder. The intensity of the signal is controlled by both the generator and the amplifier. The amplitude and frequency of the imposed fluid oscillations vary in the ranges $f=5-20 \mathrm{~Hz}$ and $b=0-7 \mathrm{~mm}$, respectively. The experiments are conducted using 2-propanol.

The motion of the oscillating meniscus in the capillary tube was first studied by a high-speed camera Basler A402k equipped with a Helios-44 M-4 lens. Depending on the experimental conditions, the frame rate was varied from 140 to $200 \mathrm{fps}$ (resolution is $800 \times 400$ pixels). Consequently, up to forty images of the meniscus could be acquired in the course of one period of oscillation. Typical data showing the meniscus location $z$ as a function of time are displayed in Fig. 2. At small and moderate amplitudes of oscillations of the acoustic louder, the liquid meniscus oscillates sinusoidally (Fig. 2a). Deviations from this 


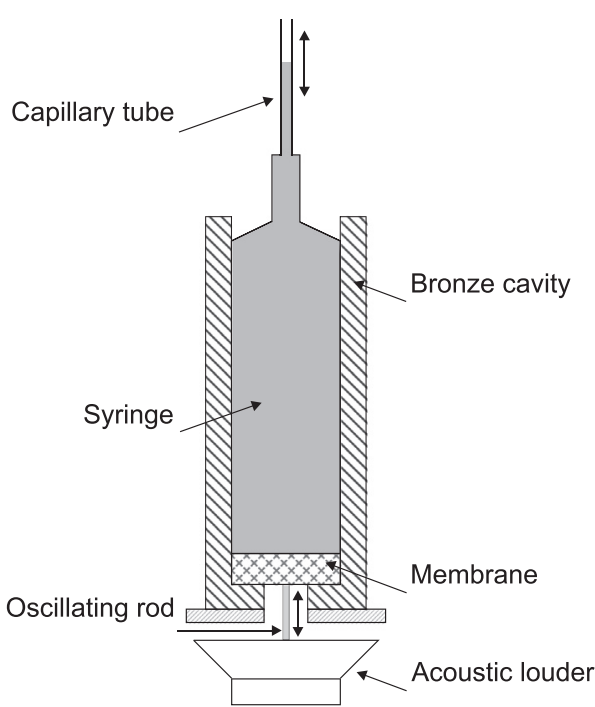

(a)

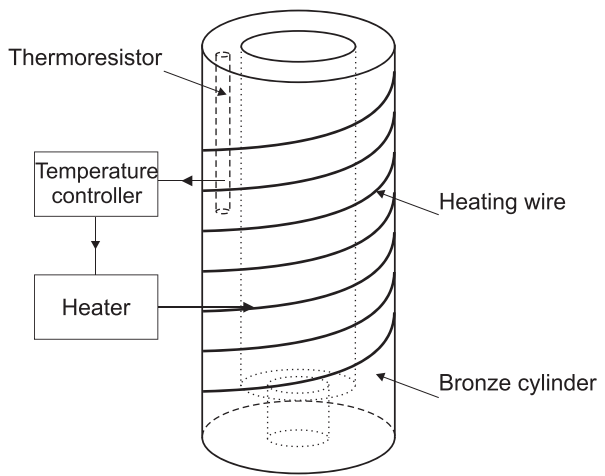

(b)

Fig. 1. (a) Sketch of the experimental setup. (b) Thermal management technique.

harmonic behaviour were observed for larger amplitudes, typically with $b>3 \mathrm{~mm}$ (Fig. 2b), and such cases were not retained in the present study.

The measurement of the diffusion rate of liquid vapour along the longitudinal axis of the capillary tube is based upon the tracking of the meniscus recession. The room ambient temperature $T_{a}$, and therefore the temperature of the fluid contained in the capillary tube is regulated by an air-conditioner system but can nonetheless vary within one Kelvin. Let us estimate the displacement of the interface between 2-propanol and air in the capillary tube with $d=1 \mathrm{~mm}$ due to a $\pm 1 \mathrm{~K}$ temperature change. The volumetric thermal expansion coefficient of 2-propanol is $V_{\beta}=0.001 \mathrm{~K}^{-1}$. Thus, the corresponding fluid volume increase for the $5 \mathrm{ml}$ syringe is $5 \times 10^{-3} \mathrm{ml}$, corresponding to a meniscus displacement up to roughly a few millimetres. This effect is significant in the experiment as the total meniscus displacement during one experiment is typically of the same order of magnitude. This explains why special care was taken in the experimental setup to control the liquid temperature within $0.1 \mathrm{~K}$ in order to limit the influence of ambient temperature fluctuations. To that end, the syringe filled with liquid is placed inside a hollow bronze cylinder, see Fig. 1b. This cavity is heated by the Joule effect generated within a uniformly coiled wire. The cavity temperature is measured by a thermoresistor and temperature management is provided by a controller Termodat $13 \mathrm{~K} 2$ which enables to maintain a fixed temperature $T$ with a $0.1 \mathrm{~K}$ accuracy.

\subsection{Experimental protocol and setup validation}

The protocol for each experiment is the following. The syringe filled with fluid is placed inside the cavity and the temperature of the controller is set to be between 1 and $2 \mathrm{~K}$ higher than the ambient temperature. Thermal equilibrium is typically reached after 30-60 min. Then the meniscus location, which is initially positioned close to the opened end of the capillary tube, is recorded with a Nikon D7000 camera. The camera is controlled by the software DcamCapture which enables to trigger image capture at regular intervals during the few hours an experiment last, typically each $15 \mathrm{~s}$. Typical images of the tube are shown in Fig. 3. As the capillary is back-illuminated by a LEDs panel, the interface between 2-propanol and air is clearly visible and its distance $z$ from the opened end of the capillary tube can be readily obtained by image processing.

To validate the setup, the evaporation kinetic without oscillations was compared to the classical result for evaporation in air of a volatile liquid contained in a circular cross-section tube, a case

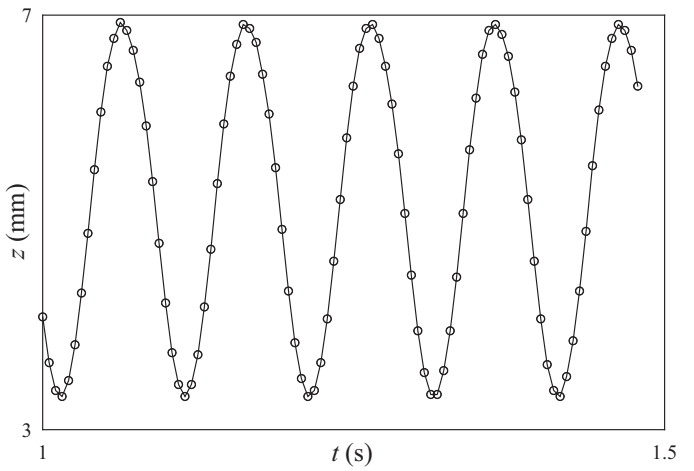

(a)

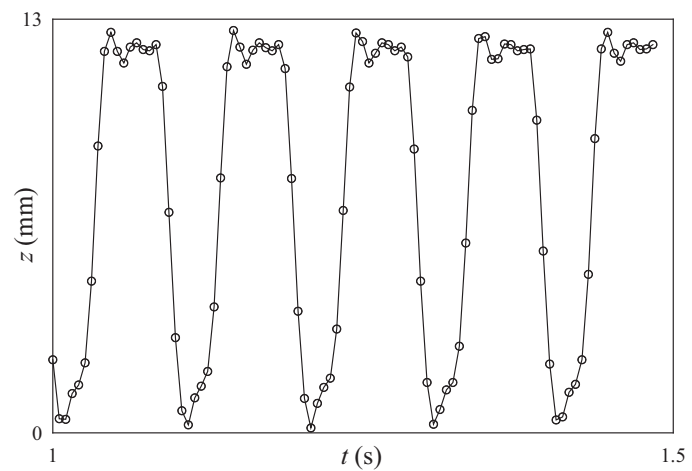

(b)

Fig. 2. Meniscus vertical location $z$ as a function of time. (a) $f=10 \mathrm{~Hz}, b=1.8 \mathrm{~mm}$. (b) $f=10 \mathrm{~Hz}, b=6.1 \mathrm{~mm}$. 


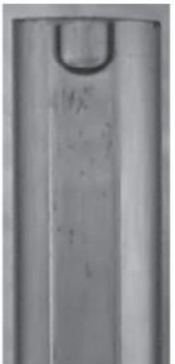

(a)

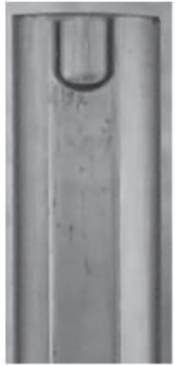

(b)

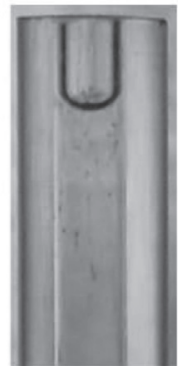

(c)

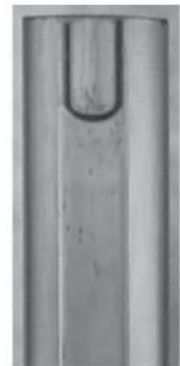

(d)
Fig. 3. Typical pictures showing the interface between 2-propanol and air in the capillary tube of diameter $1.6 \mathrm{~mm}(\mathrm{a}-\mathrm{d})$. The time interval between two successive pictures is $1500 \mathrm{~s}$.

often referred to as the Stefan diffusion tube problem, [21]. The Stefan diffusion law reads:

$z(t)=\sqrt{\frac{2 D M P_{a}}{\rho_{l} R T_{a}} \ln \left(\frac{1}{1-x_{e}}\right) t}+z_{0}$

where $D$ is the diffusion coefficient of 2-propanol in air, $M$ the molecular weight of propanol-2, $P_{a}$ the atmospheric pressure, $\rho_{l}$ the mass density of liquid 2-propanol, $R$ the ideal gas constant, $T_{a}$ the ambient temperature, $x_{e}$ the thermodynamical equilibrium mass fraction of 2-propanol in air and $z_{0}$ the meniscus position at $t=0$. In the present case, $x_{e}=0.107<1$ so that the previous equation can be simplified within a few percent accuracy into:

$z(t)=\sqrt{\frac{2 D \rho_{v}}{\rho_{l}}} t+z_{0}$

The evolution of $z$ is shown as a function of the square root of time in Fig. 4. The behaviour expected from Stefan's law, $z \sim t^{1 / 2}$, is clearly recovered. The parameter $A=\left(2 D \rho_{v} / \rho_{l}\right)^{0.5}$, which characterises the evaporation kinetic, can be obtained by fitting the experimental data to get the slope $d z / d t^{1 / 2}$. Reference data $[22,23]$ allow to calculate the expected value for coefficient $A$ at the temperature of the experiment. For the typical case shown in Fig. $4(d=1 \mathrm{~mm}, T=303 \mathrm{~K}), A=0.072 \mathrm{~mm} / \mathrm{s}^{1 / 2}$ which is in good agreement with the experimentally obtained value, $A=0.071 \mathrm{~mm} / \mathrm{s}^{1 / 2}$.

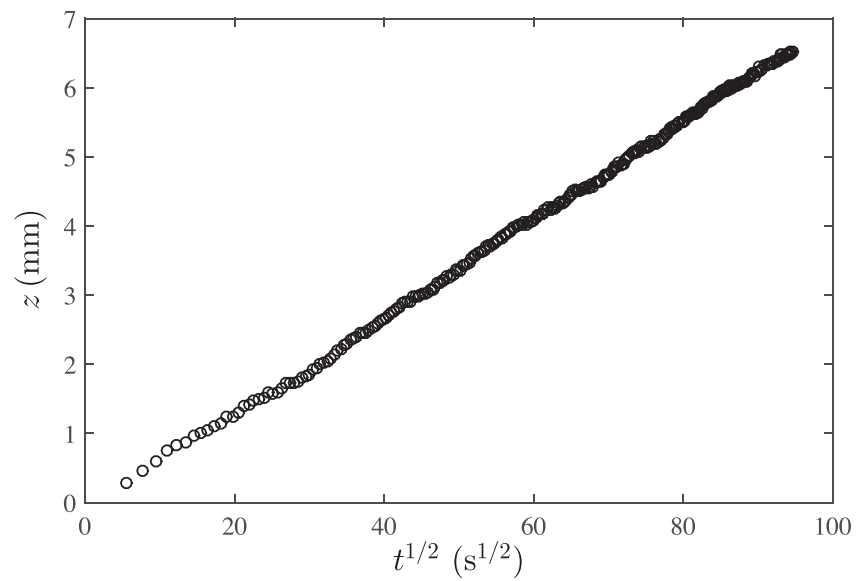

Fig. 4. Evolution of the meniscus position due to evaporation, without oscillations. The tube diameter is $d=1 \mathrm{~mm}$. The fluid temperature is regulated at $T=303 \mathrm{~K}$

\section{Results}

We now consider the case of interest in our study, where an oscillatory motion of the liquid/air interface is mechanically imposed, see Section 2.1. As already mentioned, oscillations with a radian frequency $\omega \equiv 2 \pi f$ and amplitude $b$ respectively in the range $30-125 \mathrm{~s}^{-1}$ and $0-3 \mathrm{~mm}$ are applied. The kinematic viscosity of air at room temperature is $1.5 \times 10^{-5} \mathrm{~m}^{2} / \mathrm{s}$. Thus, the largest Reynolds number reached for the oscillatory flow in the tube, $\operatorname{Re}_{\delta} \equiv 2 b \omega \delta / v$, is about $10^{2}$ and we deal with a laminar air flow above the oscillating meniscus [3].

During an experiment, the camera and the generator of harmonic signal are not synchronised and therefore images of the meniscus are captured at various phases of the liquid oscillations. As a consequence, plotting the meniscus locations as a function of time results in a "cloud" of experimental data points with sharp edges corresponding to the lowermost and uppermost locations of the meniscus, see Fig. 5. The evaporation kinetic can be divided into two stages: a short initial one (i) (up to $\sqrt{t} \approx 30 \mathrm{~s}^{1 / 2}$ for the experimental results shown in Fig. 5 second main one (ii). Noteworthy, both stages follows a diffusion like behaviour, with $z \sim t^{1 / 2}$. The initial stage lasts for relatively short period of time, 20-30 min, whereas the main regime lasts for hours. The paper focuses on this extended diffusive regime for 2-propanol vapour. It will be characterised in the following by an effective diffusion coefficient $D_{\text {eff }}$ :

$D_{e f f}=A_{e f f}^{2} \frac{\rho_{l}}{2 \rho_{v}}$

where the parameter $A_{\text {eff }}$ is obtained by fitting the experimental data.

The effect of the oscillation amplitude $b$ on $D_{\text {eff }} / D$ for different tube diameters, respectively frequency, is shown in Fig. 6(a), respectively 6(b). At a fixed frequency, $D_{\text {eff }} / D$ is found to increase with $b$ and also with the tube diameter $d$. Similarly, at a fixed $d, D_{\text {eff }} / D$ increases with $b$ and with $f$. For any capillary tube diameters and oscillations frequencies, $D_{\text {eff }} / D \rightarrow 1$ in the $b \rightarrow 0$ limit.

By analogy with Joshi et al.[12], we introduce a characteristic spatially averaged velocity of air $\langle u\rangle=b \omega / \sqrt{2}$. Therefore, the Péclet number is defined as $P e=b \omega d / \sqrt{2} D$. Fig. 7 summarises all the results for the effective, enhanced diffusion rate obtained at various parameters of liquid oscillations in different capillaries, by plotting $D_{\text {eff }} / D$ as a function of $P e$. $D_{\text {eff }} / D$ is found to be

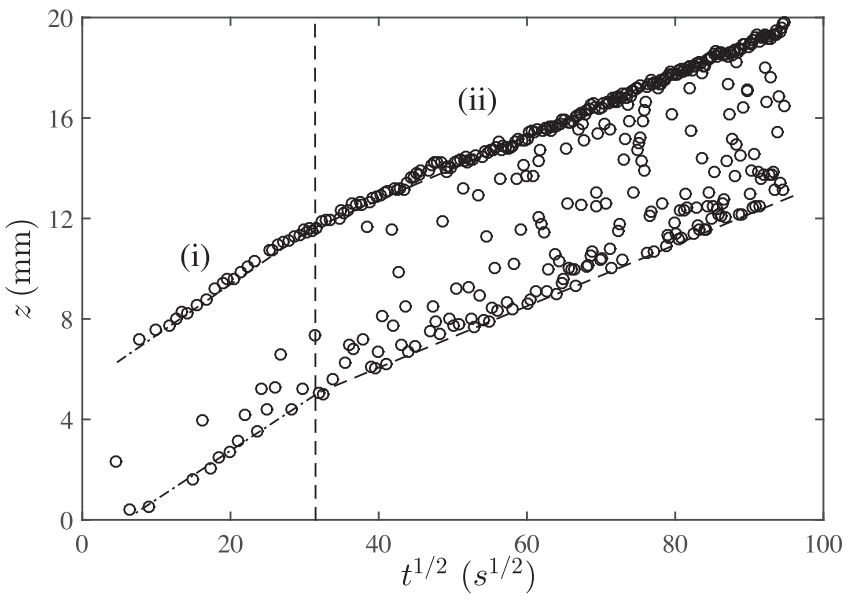

Fig. 5. Evolution of the meniscus location as a function of time when oscillations are imposed. The tube diameter $d$ is $1 \mathrm{~mm}$. Oscillations are driven at a frequency $f=$ $5 \mathrm{~Hz}$ and amplitude $b=3.4 \mathrm{~mm}$. The imposed temperature is $T=303 \mathrm{~K}$. 


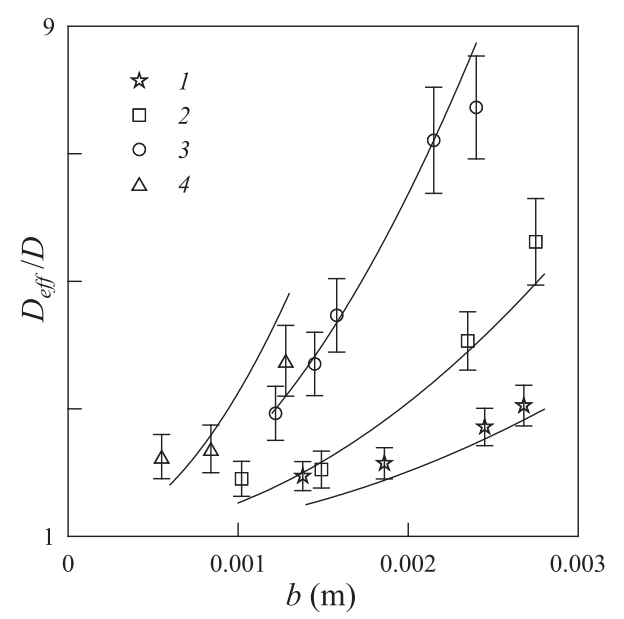

(a)

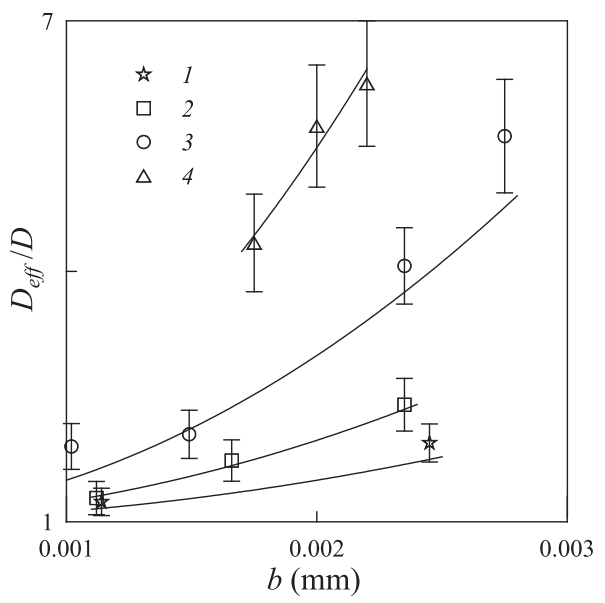

(b)

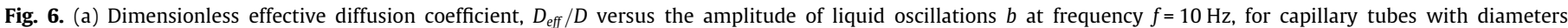
$d=0.7,1.0,1.6$ and $2.05 \mathrm{~mm}(1-4)$. (b) $D_{\text {eff }} / D$ versus $b$ in a capillary tube with $d=1.6 \mathrm{~mm}$, for frequencies $f=7,10,15$ and $20 \mathrm{~Hz}(1-4)$.

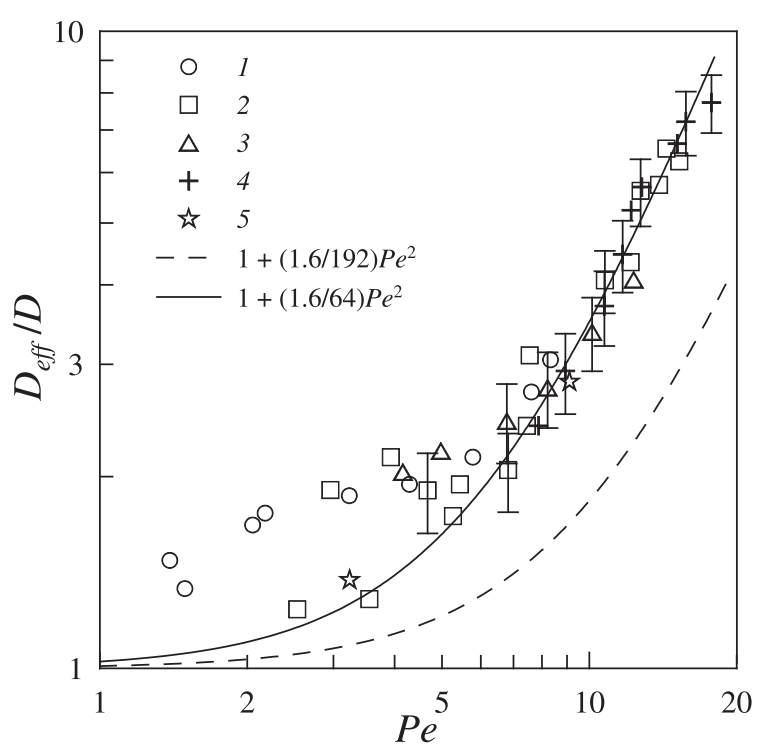

Fig. 7. Dimensionless effective diffusion coefficient, $D_{\text {eff }} / D$, is plotted versus Péclet number. Experimental data $1-5$ are obtained in capillaries with $d=0.7,1.0,1.15,1.6$ and $2.05 \mathrm{~mm}$, respectively. The dashed line corresponds to the theoretical prediction, $D_{\text {eff }} / D=1+\sigma P e^{2} / 192$, whereas the observed trend, $D_{\text {eff }} / D \approx 1+3 \times \sigma P e^{2} / 192$, is shown as a solid line.

proportional to $P e^{2}$ in the limit $P e \gg 1$. This scaling is consistent with Watson's theoretical results, as cited by Joshi et al. [12]:

$D_{\text {eff }} / D=1+f(\alpha, \sigma) P e^{2}$,

where $\sigma=v / D$ is the Schmidt number. It is worth mentioning the fact that the coefficient $f(\alpha, \sigma)$ found in the present study, where $\sigma=1.6$, is three times larger than the value predicted value for low $\alpha, f(\alpha, \sigma)=\sigma / 192$. This latter theoretical prediction, $D_{\text {eff }} / D=1+\sigma P e^{2} / 192$, is indicated as a dashed line in Fig. 7 whereas the observed trend, $D_{\text {eff }} / D \approx 1+3 \times \sigma P e^{2} / 192$, is shown as a solid line.

\section{Discussion}

In this section we comment the obtained results in the light of previous contributions of the literature, and discuss some related issues associated with the unexpectedly strong evaporation rate found in previous section.

\subsection{Comparison and discussions with previous experimental results}

To our knowledge, enhanced diffusion in laminar oscillatory flow has been studied experimentally in tubes in two earlier studies. Joshi et al. [12] injected a tracer gas (methane) at a constant infusion rate in a quartz tube where an oscillatory flow was imposed by the displacement of a motor-driven piston. They measured the spatio-temporal evolution of methane concentration along the tube using a photometric technique. Jaeger \& Kurzweg and Kurzweg et al. [11,8] studied diffusion of nitrogen contained in ambient air within rigid tubes, capillary bundles and beads packing, infused by oxygen at a constant flow rate, and in which oscillatory flow was imposed with a variable pump. It is noteworthy that, contrarily to these two experimental works, the present experimental setup does not require to impose a steady flow.

As far as the relevant dimensionless numbers are concerned, the range of Womersley numbers investigated in the present study ( $\alpha$ in the range [0.5-4]) is slightly narrower than the one used in Joshi et al. ( $\alpha$ in the range [1.5-9]) and in Kurzweg et al.'s works (where larger Womersley values, up to 25 are reported). One important difference between the present study and the aforementioned ones is that the Péclet numbers are typically much smaller in the present study. In fact, as seen in the previous Section, the Péclet number is in the range [1-20] for the present study whereas it ranges between 20 and 1000 in Joshi et al. and between 100 and 1000 in Kurzweg et al.

In Fig. 8, the results obtained in the present study are displayed together with Joshi et al. [12] and Kurzweg et al. [8] data. In this Figure, $\left(D_{\text {eff }} / D-1\right) /\left(\sigma \times P e^{2}\right) \equiv f(\alpha, \sigma) / \sigma$, see Eq. (2), is shown as a function of the Womersley number $\alpha$. This is the only possible choice to be able to extract the data points from the figures published in the previous works mentioned (for instance, obtaining the Péclet number for each data points presented in these papers is not possible, due to lack of information), taking into account the different Schmidt number $\sigma$ from one study to another (including the present one). Watson's theoretical predictions are also shown as lines, for the Schmidt number values corresponding to each of the experimental data sets, reaching the limit $1 / 192$ as $\alpha \rightarrow 0$. As already mentioned while discussing Fig. 7, Watson's theory underestimates our results by a factor 3 , whereas Joshi's results 


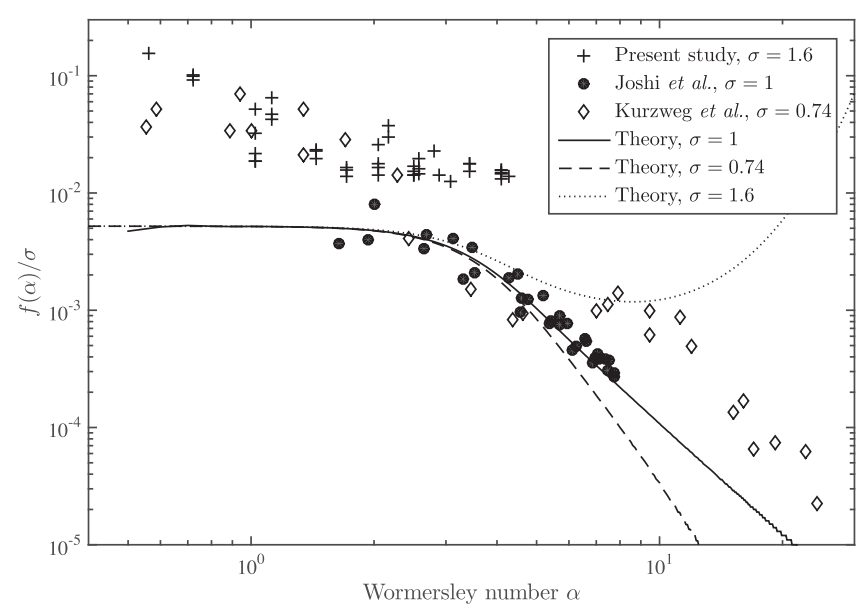

Fig. 8. $\left(D_{e} f f / D-1\right) /\left(\sigma \times P e^{2}\right)=f(\alpha, \sigma) / \sigma$ as a function of the Womersley number $\alpha$. Crosses: present study; Black circles: Joshiet al.'s data [12]; Diamonds: Kurzweg et al.'s data [8]. Lines: Watson's theoretical predictions, [6], for various values of the Schmidt number $\sigma$, see legend for details.

are in good agreement with it. The results from Kurzweg et al. are much more scattered, perhaps due to the wide range of medium used, from tubes (as in Joshi et al. and the present study) but also capillary bundle and beads packing.

\subsection{Quantification of film influence}

As mentioned in previous sections, the mechanism responsible for the increase of the evaporation rate is the coupling between transverse diffusion and longitudinal convection associated with a Taylor-dispersion transport mechanism, as developed in various contributions such as $[6,12]$. In the case of the evaporation of an oscillating meniscus, there is a possible supplementary ingredient that has not been discussed yet: the coupled transfer between the gas phase and the liquid films covering all the inner tube surface from the oscillating meniscus region. These films are driven by a capillaro-viscous coating to the tube exit where they are sucked by the capillary-disjunction pressure. Such a mechanism has first been explored in the framework of Taylor dispersion in [24] and later-on re-visited for deviation to apparent diffusive behaviour $[25,26]$. Since liquid films can capture and/or release the vapour molecules (in our case 2-propanol) in the gas, this secondary exchange could enhance the dispersion because some molecules can be trapped and later-on released into the gas, enhancing the longitudinal concentration profile. This would lead to an increase of the effective dispersion and finally contribute to enhance the evaporation rate. Following [24], we evaluate below the influence of the trap/release mechanism associated with films in our experiment. In [24], the influence on the dispersion is found from two separate contributions: (i) modifications of the tube diameter associated with the film thickness, which then changes the velocity profile and thus the dispersion coefficient, (ii) supplementary contribution associated with the trapping/release mechanisms.

(i) The condition of films on dispersion coefficient corrections due to velocity profile changes are at most expected to be of the order $O(\beta)$, where $\beta=\delta_{f} / d$ is the ratio between the film thickness and the tube diameter $d$. Since in our case $\delta_{f} \simeq 10^{-7} \mathrm{~m}$ and $D \simeq 10^{-3} \mathrm{~m}, \beta \simeq 10^{-4}$, so that such correction should be negligible in our case.

(ii) The second contribution found in [24] for the supplementary dispersion reads $\langle u\rangle E /(1+\beta)^{2}$, where $E=\langle u\rangle d^{2} \beta^{3} / 3 D_{l}(1+\beta)$ so that the correction to dispersion reads $D \times P e^{2}\left(D / D_{l}\right) \beta^{3} /$ $3(1+\beta)^{3}$, where $D_{l}$ is the self-diffusion coefficient of the evaporating gas within its liquid phase in the films. Following Eq. (2), where the correction is encapsulated within an additional Taylor-like term, the effective diffusion including Purnama's prediction for the effect of thin films evaporation would read:

$$
\left.D_{\text {eff }} / D=1+\left(f(\alpha, \sigma)+\left(D / D_{l}\right) \beta^{3} / 3(1+\beta)^{3}\right)\right) P e^{2} .
$$

Hence, it is found that this additional mechanism should add a dimensionless correction $\mathrm{Pe}^{2}\left(D / D_{l}\right) \beta^{3} /\left(3(1+\beta)^{3}\right)$ the order of magnitude of which can be found from $P e=10^{2}, D / D_{l} \simeq 10^{-2}, \beta \simeq 10^{-4}$ to be smaller than $10^{-10}$. Hence, the possible capture/release of the vapour by films is found of negligible impact on the apparent Taylor dispersion coefficient, and thus on the apparent evaporation rate in our experimental conditions. We now discuss another possible mechanism.

\subsection{Coupled stationary air down-flow with dynamic Taylor dispersion} mechanism associated with meniscus stoke

Another possible source for the increasing evaporation rate might come from a positive coupling between some existing stationary, evaporation-driven, air flow and the forced, oscillating Taylor's dispersion. As discussed in [27,21], the vapour up-flow associated with evaporation, leads to a gradient in vapour molar concentration along the tube, which, in turns also produce an air molar concentration gradient along the tube, since the total molar fraction of air and vapour everywhere equals one at equilibrium. Hence, a downward air flux driven by stationary diffusion, exists to compensate for the inward vapour, and some slow recirculation of air takes place inside the tube. Another possible source of (very small) air motion can also originates from buoyancy effects, since small air molar concentration gradient might also induce small changes in local density, thus producing a longitudinal pressure gradient [28].

One might then question the possible influence of air stationary flow on the apparent evaporation rate, when coupled with oscillating Taylor's mechanism. As discussed in classical textbook and for example in [12], Taylor's dispersion arises from the coupled effect of concentration and velocity fluctuations. Decoupling the average and the fluctuating components of the concentration and velocity fields as:

$$
\begin{aligned}
c(\mathbf{x}, t) & =\langle c\rangle(z, t)+c^{\prime}(\mathbf{x}, t), \\
u(\mathbf{x}, t) & =\langle u\rangle(z, t)+u^{\prime}(\mathbf{x}, t),
\end{aligned}
$$

where $\langle\cdot\rangle \equiv\left(4 / \pi d^{2}\right) \int_{0}^{d / 2} \cdot r d r \int_{0}^{2 \pi} d \theta$ is the spatial averaging inside the cylindrical tube, the volume species flux of vapour in the longitudinal direction then reads:

$c u-D \frac{\partial c}{\partial z}=\langle c\rangle\langle u\rangle+c^{\prime}\langle u\rangle+\langle c\rangle u^{\prime}+u^{\prime} c^{\prime}-D \frac{\partial c}{\partial z}$

and thus the average flux is:

$\left\langle q_{c}\right\rangle=\left\langle c u-D \frac{\partial c}{\partial z}\right\rangle=\langle c\rangle\langle u\rangle+\left\langle u^{\prime} c^{\prime}\right\rangle-D\left\langle\frac{\partial c}{\partial z}\right\rangle$.

Taylor's dispersion arises from the ability to show that the quadratic fluctuation term is proportional to the average concentration gradient, so that following [12], one gets:

$\left\langle u^{\prime} c^{\prime}\right\rangle-D \frac{\partial c}{\partial z}=D_{T}\left\langle\frac{\partial c}{\partial z}\right\rangle$,

such that the average flux (5) achieves an apparent effective diffusivity. 
A similar analysis can be done when an oscillating velocity is imposed. In this case, one might consider the spatio-temporal average flux of species:

$\left\langle\bar{q}_{c}\right\rangle=\langle\overline{c u}-D \overline{\overline{\partial c}}\rangle=\langle\bar{c}\rangle\langle\bar{u}\rangle+\left\langle\overline{u^{\prime} c^{\prime}}\right\rangle-D\left\langle\frac{\overline{\partial c}}{\partial z}\right\rangle$

where temporal average $=1 / T \int_{0}^{T} \cdot d t$ is also performed, so as to find a spatio temporal additional effective diffusivity from:

$\left\langle\overline{u^{\prime} c^{\prime}}\right\rangle-D\left\langle\overline{\frac{\partial c}{\partial z}}\right\rangle=D_{T}\left\langle\frac{\overline{\partial c}}{\partial z}\right\rangle$.

This result indicates that the spatio-temporal quadratic fluctuation term is the way concentration and advection are coupled to produce an effective Taylor dispersion coefficient. Hence, decomposing the fluctuations in stationary and time-varying components, we denote

$$
\begin{aligned}
& c^{\prime}(\mathbf{x}, t)=c_{0}^{\prime}(\mathbf{x})+\tilde{c}^{\prime}(\mathbf{x}) e^{i \omega t}, \\
& u^{\prime}(\mathbf{x}, t)=u_{0}^{\prime}(\mathbf{x})+\tilde{u}^{\prime}(\mathbf{x}, t) e^{i \omega t},
\end{aligned}
$$

where the stationary velocity fluctuations $u_{0}^{\prime}(\mathbf{x})$ might come from air recirculation compensating the inward vapour flow, and stationary concentration fluctuations $c_{0}^{\prime}$ from vapour molar concentration longitudinal gradient, while $\tilde{c}^{\prime}, \tilde{u}^{\prime}$ stands for the complex amplitude of sinusoidal temporal variations. It is then easy to see that

$\left\langle\overline{u^{\prime} c^{\prime}}\right\rangle=\left\langle u_{0}^{\prime} c_{0}^{\prime}\right\rangle+\left\langle\overline{\tilde{u}^{\prime} \tilde{C}^{\prime *}}\right\rangle$

where $\tilde{c}^{\prime *}$ stands the complex-conjugate of $\tilde{c}^{\prime}$. Hence, from (10) and (8), we see that the effective diffusivity can not result from a coupling between stationary flow and time varying-ones. Furthermore, the first term on the right-hand-side of (10) is precisely the one resulting from stationary terms only, that is to say the one reported in Section 2.2 when calibrating the effective average evaporation rate. Since it is much smaller that the measured effect, in the presence of oscillating meniscus, it can not explain the observed discrepancy with the apparent Taylor dispersion. Hence the possible coupling of stationary vapour molar concentration gradient with velocity oscillation can not explain the observed effect.

\subsection{Perspectives}

Since we discuss in previous paragraphs the impact of possible effects to account for the prefactor deviation from the Taylor's diffusion regime without explaining it, some perspectives are now needed. First of all, we should stress that since the temporal behaviour found for the evaporation rate is diffusive (proportional to the square root of time) and since an effective diffusion coefficient scaling as the square of the Péclet number is observed, a Taylor's dispersion mechanism clearly remains a valid explanation for the experimental observation, but for the amplitude of it.

One should bear in mind that all the proposed theoretical analysis related to some modified Taylor's dispersion rely on longitudinally invariant velocity fields. The gas velocity is obviously invariant along the $z$ direction either far from the meniscus and far from the tube opened end. But obviously, close to the meniscus, a complex three-dimensional pulsed flow occurs, either in the liquid film as well as in the gas [29]. It is expected that some recirculation related to piston-like Stokes flow [30] will affect evaporation as the concentration profile far ahead the meniscus region will be affected by these complex convection patterns. A more detailed analysis of the complex flow and transport arising nearby the meniscus is obviously out of the scope of the present paper. But it may provide a quantitative explanation for the extra evaporative flux contribution, i.e. the modified prefactor of the observed Taylor's regime.

\section{Conclusion}

The diffusion rate of alcohol vapour in capillaries under oscillations is experimentally studied. It is found that the effective coefficient for vapour diffusion is larger than the coefficient of natural diffusion: a tenfold intensification can be obtained for the largest Péclet number achieved. The effect is certainly caused by a Taylor's dispersion mechanism. Experimental results qualitatively agree with existing experimental data obtained for vapour diffusion in an oscillating flow with non-zero mean velocity, but exceed the existing theoretical predictions.

\section{Acknowledgments}

The work is supported by Ministry of Education of the Perm region (Project C26/625) and was initiated during the stay in France of one of the authors (D. Polezhaev), supported by GIP ANR (Project ANR-06-BLAN-0119-01 Intensifilm).

\section{References}

[1] G. Taylor, Dispersion of soluble matter in solvent flowing slowly through a tube, Proc. R. Soc. Lond. Ser. A. Math. Phys. Sci. 219 (1137) (1953) 186-203.

[2] R. Aris, On the dispersion of a solute in a fluid flowing through a tube, Proc. R. Soc. Lond. Ser. A. Math. Physi. Sci. 235 (1200) (1956) 67-77.

[3] D.M. Eckmann, J.B. Grotberg, Experiments on transition to turbulence in oscillatory pipe flow, J. Fluid Mech. 222 (1991) 329-350.

[4] R. Aris, On the dispersion of a solute in pulsating flow through a tube, Proc. R. Soc. Lond. Ser. A. Math. Phys. Sci. 259 (1298) (1960) 370-376.

[5] P.C. Chatwin, On the longitudinal dispersion of passive contaminant in oscillatory flows in tubes, J. Fluid Mech. 71 (03) (1975) 513-527.

[6] E.J. Watson, Diffusion in oscillatory pipe flow, J. Fluid Mech. 133 (1983) 233244.

[7] U.H. Kurzweg, L.D. Zhao, Heat transfer by high-frequency oscillations: a new hydrodynamic technique for achieving large effective thermal conductivities, Phys. Fluids 27 (11) (1984) 2624.

[8] U.H. Kurzweg, G. Howell, M.J. Jaeger, Enhanced dispersion in oscillatory flows, Phys. Fluids 27 (5) (1984) 1046.

[9] U.H. Kurzweg, Enhanced heat conduction in osillating viscous flows within parallel-plate channels, J. Fluid Mech. 156 (1985) 291-300.

[10] C.-O. Ng, A time-varying diffusivity model for shear dispersion in oscillatory channel flow, Fluid Dyn. Res. 34 (6) (2004) 335-355.

[11] U.H. Kurzweg, M.J. Jaeger, Determination of the longitudinal dispersion coefficient in flows subjected to high-frequency oscillations, Phys. Fluids 26 (6) (1983) 1380

[12] C.H. Joshi, R.D. Kamm, J.M. Drazen, A.S. Slutsky, An experimental study of gas exchange in laminar oscillatory flow, J. Fluid Mech. 133 (1983) 245-254.

[13] U. Akdag, M. Ozdemir, A.F. Ozguc, Heat removal from oscillating flow in a vertical annular channel, Heat Mass Transfer 44 (4) (2008) 393-400.

[14] U.H. Kurzweg, Enhanced heat-conduction in fluids subjected to sinusoidal oscillations, J. Heat Trans-T. ASME 107 (2) (1985) 459-462.

[15] J.G. Zhang, U.H. Kurzweg, Numerical simulation of time-dependent heat transfer in oscillating pipe flow, J. Thermophys. Heat Transfer 5 (3) (1991) 401-406.

[16] D.Y. Lee, S.J. Park, S.T. Ro, Heat transfer in the thermally developing region of a laminar oscillating pipe flow, Cryogenics 38 (6) (1998) 585-594.

[17] P.P. Ma, X.M. Li, D.N. Ku, Heat and mass-transfer in separated flow region for high prandtl and schmidt numbers under pulsatile conditions, Int. J. Heat Mass. Transfer 37 (17) (1994) 2723-2736.

[18] W. Cooper, V. Nee, K.T. Yang, Fluid mechanics of oscillatory and modulated flows and associated applications in heat and mass transfer: a review, J. Energy, Heat Mass Transfer 15 (1993) 1-19.

[19] T. S. Zhao, P. Cheng, Heat transfer in osillatory flows, in: T.S. Zhao, P. Cheng (Ed.), Annual Review of Heat Transfer, IX, 1998, Ch. 7

[20] Z. Yu, X. Mao, A.J. Jaworski, Experimental study of heat transfer in oscillatory gas flow inside a parallel-plate channel with imposed axial temperature gradient, Int J. Heat. Mass. Transfer 77 (2014) 1023-1032.

[21] R.B. Bird, W.E. Stewart, E.N. Lightfoot, Transport Phenomena, John Wiley \& Sons, 2007.

[22] D.W. Pepper, D. Carrington, Modeling Indoor Air Pollution, World Scientific Publishing, 2009.

[23] V. Rabinovich, Z.Y. Khavin, Handbook of Chemistry, Khimiya, 1991. 
[24] A. Purnama, The effect of dead zones on longitudinal dispersion in streams, J Fluid Mech. 186 (1988) 351-377.

[25] C.G. Phillips, S.R. Kaye, C.D. Robinson, Time-dependent transport by convection and diffusion with exchange between two phases, J. Fluid Mech. $297(-1)(1995) 373$.

[26] C.G. Phillips, S.R. Kaye, Approximate solutions for developing shear dispersion with exchange between phases, J. Fluid Mech. 374 (1998) 195-219.

[27] S. Chauvet, Effet des Films Liquides en Ât'evaporation (Ph.D. thesis), Toulouse University, 2009.
[28] M. Emin Erdogan, P.C. Chatwin, The effects of curvature and buoyancy on the laminar dispersion of solute in a horizontal tube, J. Fluid Mech. 29 (1967) 465484.

[29] H. Gelderblom, H.A. Stone, J.H. Snoeijer, Stokes flow in a drop evaporating from a liquid subphase, Phys. Fluid. 25 (10) (2013). 102102-102102-15.

[30] F. Katopodes, A. Davis, H. Stone, Piston flow in a two-dimensional channel, Phys. Fluids 12 (5) (2000) 1240-1243. 